\title{
A novel unsupervised Levy flight particle swarm optimization (ULPSO) method for multispectral remote sensing image classification
}

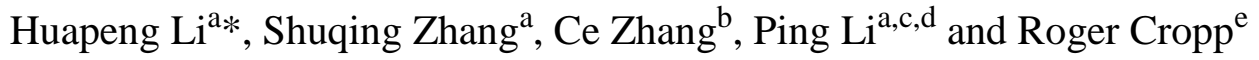 \\ ${ }^{a}$ Northeast Institute of Geography and Agroecology, Chinese Academy of Sciences, \\ Changchun, 130012, China; ${ }^{b}$ Lancaster Environment Centre, Lancaster University, Lancaster \\ LA1 2YQ, UK; ${ }^{c}$ University of Chinese Academy of Sciences, Beijing, 100049, China; \\ ${ }^{d}$ Urban Development Institute, Jilin Academy of Social Sciences, Changchun, 130000, China; \\ ${ }^{e}$ Griffith School of Environment, Griffith University, Gold Coast, 4222, Australia \\ *Corresponding author. Email: lihuapeng@ neigae.ac.cn
}

The rapid development of earth observation technology has produced large quantities of remote sensing data. Unsupervised classification (i.e. clustering) of remote sensing image, an important means to acquire land use/cover information, has become increasingly in demand due to its simplicity and ease of application. Traditional methods such as $k$-means struggle to solve this NP-hard image classification problem. Particle swarm optimization (PSO), always achieving better result than $k$-means, has recently been applied to unsupervised image classification. However, PSO was also found to be easily trapped on local optima. This paper proposes a novel unsupervised Levy flight particle swarm optimization (ULPSO) method for image classification with a balanced and powerful searching capability. This results from a new searching strategy: the worst particle in the swarm is targeted and its position is updated with Levy flight at each iteration. The effectiveness of the proposed method is tested with three types of remote sensing imagery (Landsat TM, FLC, and QuickBird) that are distinct in terms of spatial and spectral resolution, and landscape. Our results show that ULPSO is able to achieve significantly better and more stable classification results than $k$-means and the other two intelligent methods based on genetic algorithm (GA) and particle swarm optimization (PSO) over all of the experiments. ULPSO is, therefore, recommended as an effective alternative for remote sensing image classification.

\section{Introduction}

Land use/cover data, reflecting the basic natural and social processes, plays an important role in the earth sciences (Loveland et al. 2000; de Colstoun et al. 2006; Huang and Laffan 2009; Deng et al. 2015). It serves as the basis for a variety of predictive models (e.g. ecosystem, hydrologic, and atmospheric models) that simulate the functioning of the Earth system (Bounoua et al. 2002; Jung et al. 2006; Verburg et al. 2011). Furthermore, land use/cover data is essential to understand the complex interactions between human activities and global change (Bonan, 1997; Gong et al. 2013). Currently, it is widely accepted that remote sensing is the mainstream means to produce land cover data because of its many advantages over fieldwork including cost-effectiveness, instantaneous measurement, synoptic view, and high 
multi-temporal coverage ( $\mathrm{Li}$ et al. 2016b). In fact, classifying remote sensing image to available land cover data is regarded as one of the core tasks of the remote sensing community (Li et al. 2011; Wilkinson 2005).

Generally speaking, there are three basic learning approaches to image classification, namely supervised, semi-supervised and unsupervised. Although supervised methods generally produce accurate results, they involve a significant amount of human effort for training sample collection (Duda and Canty 2002). Semi-supervised methods utilize a relatively small amount of labeled data to aid unsupervised training for image classification (Camps-Valls et al. 2007). In other words, training samples, whose acquisition is labour-intensive and time consuming, are required either in the supervised or in the semi-supervised. However, along with the fast development of earth observation technology, remote sensing data set grows rapidly with massive quantities of data currently archived, thus posing a great challenge for the two types of approaches. In contrast, unsupervised (i.e. clustering) methods are in no need of training samples, thereby dawning increasingly attention in the remote sensing community (Yu et al. 2012; Xu et al. 2013). Two commonly used unsupervised methods, $k$-means and fuzzy c-means (FCM), in which simple iterative rules are employed, generally converge rapidly ( $\mathrm{Li}$ et al. 2016a). However, they often have difficulty reaching the global optimal solution (Bandyopadhyay and Maulik 2002; Yildirim 2014).

Unsupervised image classification can be transformed into an optimization problem, to which artificial intelligent algorithms could help. Consequently methods such as genetic algorithm (Maulik and Bandyopadhyay 2000) and artificial immune systems (Zhong et al. 2006) have been employed for image classification. Recently, a newly proposed nature-inspired algorithm, particle swarm optimization (PSO), has become popular because of its impressive performance when solving a broad range of optimization problems such as function optimization (Seo et al. 2006), flow-shop scheduling (Tseng et al. 2008), and image processing (Chang et al. 2009). The feasibility of PSO in solving the complex remote sensing image classification has also been demonstrated (Omran et al. 2005; Wong et al. 2011; Mukhopadhyay et al. 2015). However, some studies found that PSO is more likely to become trapped on local optima when solving large-scale complex problems (like multi-peak-searches) (Das et al. 2016), such as the image clustering problem (Li et al. 2016b). Currently, the global best solution is employed in the evolution process of PSO (called "elitism strategy") to accelerate the convergence of PSO. However, this might cause the swarm to converge prematurely (i.e. on local optima) since population diversity decreases with the evolution of particles (Yu et al. 2013), leading to a local optimum without guaranteeing global convergence (Yang 2010).

Some efforts have been made to improve the performance of PSO-based image clustering. For example, Xu and Zhang (2009) developed a fuzzy PSO method for image clustering. Paoli et al. (2009) clustered hyperspectral image by using multiobjective PSO. Yang et al. (2009) presented a hybrid clustering algorithm based on PSO and K-harmonic means. Wang et al. (2012) employed PSO to increase the accuracy of sub-pixel mapping created by a sub-pixel/pixel spatial attraction model (SPSAM). Naeini et al. (2014) integrated PSO with decision analysis to improve the dynamic clustering of image. Most of the current studies, however, just combined other algorithms or classifiers with PSO in hope of gaining a better 
clustering result, whereas few efforts have been dedicated to the improvement of PSO from a standpoint of searching mechanism. Such a fundamental innovation of PSO would benefit its applications not only in image clustering, but also in the other fields.

In this paper, a novel unsupervised Levy flight particle swarm optimization (ULPSO) method is proposed for remote sensing image classification. The main contribution of this work is the establishment of a new searching mechanism for PSO by using Levy flight, which is a class of random walk employed by some animals when foraging foods (Edwards et al. 2007). In theory, Levy flight with various step lengths exhibiting non-Gaussian distributions could maintain the level of population diversity of standard PSO, thus improving the likelihood of approaching (or reaching) the optimal solution to the classification problem. To the best of our knowledge, this is the first application of Levy flight to improve the swarm intelligence based image classification. The proposed method is tested with three kinds of multispectral images (Landsat TM, FLC, and QuickBird) that are different in terms of spatial and spectral resolution, and landscape. The experiments results have demonstrated the superiority of the proposed method.

The remainder of this paper is organized as follows. In Section 2, the PSO and Levy flight are briefly outlined; the proposed ULPSO method is described in detail in Section 3; in Section 4, the experiments and results are provided; and discussion and conclusions are made in Sections 5 and 6, respectively.

\section{Particle swarm optimization and Levy flight}

\subsection{Particle swarm optimization}

PSO, a meta-heuristic optimization algorithm (Kennedy and Eberhart 1995), is inspired by the behaviours of bird flocking and fish schooling in the process of food searching. It acquires intelligence through mutual communication and cooperation among the individuals (Paoli et al. 2009). For PSO, a particle of swarm, denoting a candidate solution to the optimization problem, is initially assigned by a random position within the search space; the particle then strives to move towards the promising positions according to its own experience and the experience from neighbour particles, in other words, the particle updates positions by tracking its personal best position (pbest) and the global best position (gbest) memorized in each iteration (Masoomi et al. 2013); by doing so, the whole swarm gradually moves to the promising areas, thus approaching (or achieving) the optimal solution in the end.

Figure 1 is here

Figure 1 illustrates the evolution process of a particle in a 2-D spatial space (Liu et al. 2008). In the figure, $X_{i}(t)$ and $V_{i}(t)$, respectively, denote the position and velocity of the $i$-th particle at time $t, \quad X_{i}^{\text {pbest }}$ and $X_{\text {gbest }}$ are the best positions found by the $i$-th particle and the whole swarm, respectively. Two velocities $V_{i}^{\text {pbest }}$ and $V^{\text {gbest }}$ pointing to $X_{i}^{\text {pbest }}$ and $X_{\text {gbest }}$, respectively, are then combined with $V_{i}(t)$ to generate the velocity $V_{i}(t+1)$ of the particle at time $t+1$. The particle then moves to its new position $X_{i}(t+1)$ with $V_{i}(t+1)$, which is closer to the global optimal solution. A detailed calculation of velocity and position of particle can be found in Section 3. By iterating the 
above process, the particle swarm could progressively approach the global optimum.

\subsection{Levy flight}

It is widely accepted that randomization exerts an important effect on both exploration and exploitation of meta-heuristic algorithm. The essence of such randomization is the random walk, a process consisting of taking a number of consecutive random steps (Yang 2010). Brownian random walks, obeying a Gaussian distribution, are the most popular walks in nature. Specifically, Levy flight, a class of random walk, has the potential to maximize the efficiency of resource searching in uncertain environments (Nayak et al. 2009). Thus, it has been employed by many animals when foraging foods, such as albatrosses and fruit flies, and spider monkeys (Edwards et al. 2007). As a matter of fact, some previous efforts found that Levy flight could improve the performance of nature-inspired algorithms (Senthilnath et al. 2013; Senthilnath et al. 2016).

The step length $(S)$ of Levy flight is drawn from the Levy distribution $(L(S))$, a simple power-law formula $L(S) \sim|S|^{-1-\beta}$, where $0<\beta \leq 2$ is an index controlling the shape of the distribution. Mathematically, a Levy distribution can be simply defined as (Yang and Deb 2013):

$$
L(s, \gamma, \mu)=\left\{\begin{array}{ll}
\sqrt{\frac{\gamma}{2 \pi}} \exp \left[-\frac{\gamma}{2(s-\mu)}\right] \frac{1}{(s-\mu)^{3 / 2}}, & 0<\mu<s<\infty \\
0 & \text { otherwise }
\end{array},\right.
$$

where $\mu$ is the location parameter, and $\gamma$ is a scale parameter controlling the scale of distribution.

Levy distribution can also be described in terms of Fourier transformation as shown in Equation (2) (Yang 2010):

$$
F(k)=\exp \left[-\alpha|k|^{\beta}\right], 0<\beta \leq 2,
$$

where $\alpha$ and $\beta$ determine the distribution of the Fourier transformation. Specifically, $\alpha \quad(\in[-1,1])$ is a scale parameter (known as skewness or scale factor) indicating the skew direction; $\beta$ is a parameter that controls the shape of the probability distribution within $(0,2]$ : the smaller the $\beta$ is, the longer the possible jump is.

Since the analytic form of the integral is unobtainable for general $\beta$, a few special cases, instead, were usually employed in real applications. In particular, for $\beta=1$ and $\beta=2$, the Fourier transform corresponds to a Cauchy distribution $(F(k)=\exp [-\alpha|k|])$ and a Gaussian distribution $\left(F(k)=\exp \left[-\alpha k^{2}\right]\right)$, respectively.

In fact, Levy stochastic process with diverging moments can be characterized by probability density. Once the density is given, the step length $(S)$ of Levy flight can then be estimated.

\section{Unsupervised Levy flight particle swarm optimization method}

The ULPSO method was developed from PSO and Levy flight for the purpose of unsupervised remote sensing image classification. Suppose a remote sensing image consists of $N$ pixels with $D$ attributes and $K$ classes for classification. In principle, ULPSO 
classifies the image by searching for a fixed number $(K)$ of optimal cluster centres $\left(C_{1}, C_{2}, \ldots, C_{K}\right)$ within $\mathfrak{R}^{D}$, so that the clustering metric $(M)$, i.e. the sum of the Euclidean distances from the pixels to their respective cluster centres (Maulik and Bandyopadhya 2000), can be minimized. The clustering metric can be mathematically denoted as follows:

$$
M=\sum_{i=1}^{K} \sum_{X_{j} \in C_{i}}\left\|X_{j}-Z_{i}\right\|,
$$

where $x_{j}$ is an arbitrary pixel of the image belonging to class $i(i=1,2, \ldots, K)$, with $Z_{i}$ as its cluster centre, and $j$ is the pixel number of class $i$.

Figure 2 shows the flowchart of ULPSO, in which the main procedures including particle position initialization, fitness evaluation, particle position searching, pbest and gbest updating, and position searching by Levy flight are detailed in the next few sections.

Figure 2 is here

\subsection{Particle representation and initialization}

In PSO, a particle, denoting a candidate solution to the problem of clustering centre optimization, is formed by connecting the cluster centre of each class represented with a sequence of real numbers. The length of the particle is $D \times K$ ( $K$, the number of classes), where the first $D$ positions represent the cluster centre of class one, the second $D$ positions represent that of class two, and so on. Suppose the number of released particles (Npop) is $n$, at the beginning of iteration, each particle of the swarm is randomly assigned a position, which can be generated as follows:

$$
X_{i}^{j}=X_{\min }^{j}+r\left(X_{\max }^{j}-X_{\min }^{j}\right),
$$

where $X_{i}^{j}$ is the position at the $j$-th attribute for the $i$-th particle, $X_{\min }^{j}$ and $X_{\max }^{j}$ are the minimum and maximum values of the $j$-th attribute, respectively, and $r$ is drawn from a uniform distribution $[0,1]$.

\subsection{Fitness evaluation}

The clustering metric ( $M$ ) is inversely proportional to the quality of clustering, i.e. the lower the value of cluster metric, the better the quality of the clustering, since pixels in the same cluster should be as close (i.e. have high similarity) as possible ( $\mathrm{Li}$ et al. 2016a). Thus, the fitness function is defined on the basis of clustering metric as follows:

$$
\text { fit }=1 /(M+1) \text {, }
$$

where $M$ is calculated using Equation (3).

The fitness of a single particle at each iteration is calculated using Equation (5). For the $i$-th particle, its fitness ( $\left.f i t_{i}^{\text {pbest }}\right)$ as well as the position $\left(X_{i}^{\text {pbest }}\right)$ are then memorized. In addition, the global best fitness of the whole swarm ( $f i t^{\text {gbest }}$ ) and its corresponding position $\left(X^{\text {gbest }}\right)$ are also identified and recorded, respectively.

\subsection{Optimal position searching of particle}


After having assessed the fitness, the velocity of each particle is updated using the following equation:

$$
V_{i}(t+1)=w V_{i}+c_{1} r_{1}\left(X_{i}^{\text {pbest }}-X_{i}(t)\right)+c_{2} r_{2}\left(X^{\text {gbest }}-X_{i}(t)\right),
$$

where $V_{i}(t+1)$ and $V_{i}(t)$ are the velocities of the $i$-th particle at time $t+1$ and $t$, respectively; $X_{i}^{\text {pbest }}$ and $X_{i}(t)$ are the historical best position and the position at time $t$ for the $i$-th particle, respectively; $X^{\text {gbest }}$ is the historical best position of the whole swarm; $r_{1}$ and $r_{2}$ are two uniformly distributed random numbers in the range $[0,1] ; c_{1}$ and $c_{2}$ are acceleration coefficients; $W$ is the inertia weight determining the effect of velocity at time $t$ on the current (at time $t+1$ ) velocity.

Accordingly, the position of each particle is involved with the newly generated velocity (Figure 1):

$$
X_{i}(t+1)=X_{i}(t)+V_{i}(t+1)
$$

where $X_{i}(t+1)$ and $X_{i}(t)$ are the positions of the $i$-th particle at time $t+1$ and $t$, respectively.

\subsection{Updating pbest and gbest}

When all particles of the swarm having finished their movements, the corresponding fitness values are recalculated using Equation (5). The personal best position for the $i$-th particle is then updated in the following way:

$$
X_{i}^{\text {pbest }}=\left\{\begin{array}{ll}
X_{i}(t+1), & \text { fit } t_{i}^{\text {pbest }}<\text { fit }_{i}(t+1) \\
X_{i}^{\text {pbest }} & \text { otherwise }
\end{array},\right.
$$

where $f_{i} t_{i}(t+1)$ denotes the fitness of the $i$-th particle at time $t+1$.

The global best position can be assigned as follows:

$$
X^{\text {gbest }}=\left\{\begin{array}{ll}
X^{\text {gbest }}(t+1), & \text { fit } t^{\text {gbest }}<f i t^{\text {gbest }}(t+1) \\
X^{\text {gbest }} & \text { otherwise }
\end{array},\right.
$$

where $f_{i t}^{\text {gbest }}(t+1)$ denotes the best fitness of the whole swarm at time $t+1$, with $X^{\text {gbest }}(t+1)$ as the corresponding position.

\subsection{Global searching by Levy flight}

As mentioned before, Levy flight is more efficient than Brownian random walks when exploring in a large-scale search space. There are several ways of simulating Levy stochastic process, and one of the most efficient ways is to employ the Mantegna algorithm (Mantegna 1994), in which the step length $S$ of Levy flight can be calculated as follows:

$$
s=0.01 \frac{\mu}{|v|^{\frac{1}{\beta}}} \lambda,
$$

where the factor 0.01 comes from the fact that $L / 100(L=1)$ is the typical step size of 
walks/flights (Yang 2010), where $L$ is the typical length scale; $\mu, v$ and $\lambda$ are, respectively, drawn from normal distributions:

$$
\mu \sim N\left(0, \sigma_{\mu}^{2}\right), v \sim N\left(0, \sigma_{v}^{2}\right), \lambda \sim N(0,1),
$$

where

$$
\sigma_{\mu}=\left[\frac{\Gamma(1+\beta) \sin \left(\pi \frac{\beta}{2}\right)}{\Gamma\left(\frac{(1+\beta)}{2}\right) \beta 2^{\frac{(\beta-1)}{2}}}\right]^{\frac{1}{\beta}}, \sigma_{v}=1,
$$

where $\Gamma$ is the gamma function, and $\beta$ is a constant varying between 1 and 2 .

Global searching can be initiated by exploring new search areas. To enhance the swarm's exploration capacity, the worst particle (i.e. the particle with the lowest fitness) at the current iteration is targeted and its position is then updated with Levy flight (step $S$ ):

$$
X_{i}^{\text {new }}=X_{i}+S,
$$

where $X_{i}^{\text {new }}$ denotes the newly position for the $i$-th particle (the worst particle), whose original position is $X_{i}$.

The fitness of the updated particle is subsequently recalculated.

\subsection{Stopping condition}

The search iteration stops only when the number of maximum iterations (Miter) is reached. In this case, the optimal position, i.e. a group of cluster centres as the solution to the unsupervised classification problem, is acquired. The image is then classified on the basis of the solution.

\subsection{Pseudocode of ULPSO}

Initialize the control parameters (Npop, Miter, $w, c_{1}$, and $c_{2}$ )

Randomly initialize the positions of particles using Equation (4)

Evaluate the fitness of particles using Equation (5)

Memorize the gbest and pbest of particles

while iter< Miter do

for $i=1$ : Npop

Update the velocity of each particle using Equation (6)

Update the position of each particle using Equation (7)

end for

Evaluate the fitness of new particles using Equation (5)

for $i=1$ : Npop

Update pbest using Equation (8)

end for

Update gbest using Equation (9)

Identify the particle with the lowest fitness

Update the particle position with Levy flight using Equation (13)

Update the fitness of the updated particle using Equation (5) 


$$
\text { iter }=\text { iter }+1
$$

end while

Output the optimal solution

\section{Experiments and analysis}

The effectiveness of the proposed method was tested with three kinds of remote sensing images, namely Landsat TM, FLC, and QuickBird. In addition, traditional $k$-means as well as two intelligent unsupervised classification methods based on standard genetic algorithm (denoted as UGA) and particle swarm optimization (denoted as UPSO), respectively, were also implemented for accuracy comparison (Srinivasan and Seow 2003). Note, an experiment denotes the classification of a particular imagery with the four methods ( $k$-means, UGA, UPSO, and ULPSO), e.g. QuickBird experiment.

The number of image clustering can be either predefined or estimated during iteration (i.e. auto-clustering). In an auto-clustering method, varied numbers of clusters, instead of a fixed one, are usually produced during iterations (Maulik and Saha 2010). The numbers of variables to be optimized may thus vary for different classification methods, leading to an incomparable classification environment. Therefore, the number of clusters is uniformly assigned for all of the methods in each experiment.

To evaluate the performance of methods quantitatively, some commonly used measures of classification accuracy were calculated for each classification, namely overall accuracy (OA), producer's accuracy (PA), user's accuracy (UA), and Kappa coefficient (KA). For detailed information on how to calculate these measures, readers are reference to Foody et al. (2002).

\subsection{Data sets}

Landsat TM: The imagery (Row/Path: 120/27) was collected by Landsat Thematic Mapper on August 27, 2007. A subset of the image over Zhalong National Nature Reserve (ZNNR) of China was chosen as our test image (Figure 3). It is of size $310 \times 310$ pixels with six spectral bands (bands 1-5 and band 7). The spectral wavelengths vary from 0.45 to $2.35 \mu \mathrm{m}$. The spatial of the image is $30 \mathrm{~m}$. It has a typical heterogeneous landscape, integrated by natural wetland and anthropic farmland. In reference to the field work, five classes including marsh, meadow, farmland, saline land, and water were identified for the image. A total of 7607 samples (see Figure 3(b)) were gathered for testing the image classification results.

FLC: The image was acquired by the M7 scanner over Tippecanoe County, Indiana, US, in June 1966 (Tadjudin and Landgrebe, 2000). A subset of the original image with a size of $100 \times 118$ pixels and 12 multispectral bands was employed in this experiment. The spectral wavelengths of bands range from 0.40 to $1.00 \mu \mathrm{m}$. It is of a typical farmland landscape consisting of four classes: soybeans, rye, red clover, and wheat. A total of 7936 samples were available along with the image for accuracy assessment (Figure 4(b)).

QuickBird: The image was gathered over the Yalvhe farm, China, on September 4, 2005. The image provides $2.4 \mathrm{~m}$ spatial resolution in four multispectral bands with the spectral wavelength ranging from $0.45-0.90 \mu \mathrm{m}$ (Wang et al. 2004). A subset of the image with a size of $350 \times 350$ pixels, covering mostly farmland and woodland, was chosen for this experiment. According to the field investigation, the image is mainly covered by five classes, namely paddy field, farmland, grass, forest, and shadow. A total of 5781 samples were collected to 
evaluate the classification results of the image (Figure 5(b)).

\subsection{Parameters initialization}

To make a fair comparison, the same values, i.e. the maximum iteration number of 1000 and population size of 40 , were assigned for the common parameters of the three intelligent methods (UGA, UPSO, and ULPSO). Other parameters for each of the methods were provided as follows: for UGA, crossover rate $p_{c}=0.8$, mutation rate $p_{m}=0.01$, generation gap $p_{S}=0.9$ (Karaboga and Akay 2009); for UPSO and ULPSO, acceleration coefficients $c_{1}=c_{2}=1.8$, inertia weight $W=0.6$ (Vesterstrom and Thomsen 2004). As a benchmark, traditional $k$-means was also implemented with control parameters: the maximum iteration number $=1000$, the pixel change threshold $=0 \%$; the classification number assigned to the three images was five, four, and five, respectively. It is noted that candidate solutions of the UGA, UPSO, and $k$-means were initialized in the same way as ULPSO (i.e. by using Equation (4)).

\subsection{Classification Results}

Figures 3, 4 and 5 show the land-cover classification results of the three experiments. The corresponding classification accuracies including PA, UA, and OA achieved by the four methods ( $k$-means, UGA, UPSO, and ULPSO) are summarized in Tables 1-3, respectively. Table 4 provides the Kappa $Z$-test results for the classifications. In general, ULPSO outperformed the other methods ( $k$-means, UGA, and UPSO) over all three experiments. The overall accuracy achieved by ULPSO was higher than $k$-means, UGA, and UPSO by $19.01 \%$, $11.80 \%$, and $11.31 \%$, respectively, for the TM experiment, and by $25.62 \%, 6.13 \%$, and $1.12 \%$, respectively, for the FLC experiment. For the QuickBird experiment, the improvements were $8.67 \%, 5.83 \%$, and $0.83 \%$, respectively (Table 3 ).

Figures 3-5 are here

For the TM experiment, a large part of marsh areas were misidentified as farmland in the $k$-means classification (Figure 3 (c)), resulting in low accuracies (both PA and UA) of both marsh and farmland (Table 1). The results of the three intelligent methods (UGA, UPSO, and ULPSO) were more accurate than $k$-means because of better differentiations between marsh and farmland (Figure 3). However, both UGA and UPSO tended to overestimate the marsh class, thus leading to a low UA (lower than 62\%) for the class. Fortunately, the proposed ULPSO could better identify the marsh class (UA > 82\%), thereby providing the best classification accuracy. The Kappa Z-test further indicated that ULPSO performed significantly better than $k$-means, UGA, and UPSO (Table 4).

Tables 1-4 are here

For the FLC experiment, the performance of $k$-means was also very poor, which misclassified a majority of wheat as soybeans (Figure 4 (c)). UGA notably improved the identification of wheat, but mislabeled some rye pixels as wheat (Figure 4 (d)). As a result, a 
low PA for wheat $(<76 \%)$ and UA for rye $(<80 \%)$ were produced (Table 2$)$. In comparison, satisfactory results (OA > 95\%) were yielded by UPSO and ULPSO, which relatively well separated the four classes (Figure $4(\mathrm{e}, \mathrm{f})$ ). Yet, ULPSO still outperformed UPSO in this experiment due to its better discrimination between wheat and rye (Figure 4 (f)). Thus, similar to the TM experiment, ULPSO achieved statistically significantly better result than $k$-means, UGA, and UPSO (Table 4).

As for the QuickBird experiment, $k$-means and UGA produced lower but comparable classification results (with OA around 65\%). In the $k$-means classification, only a small part of forest was correctly identified (Figure 5 (c)), leading to an extraordinarily low PA for forest $(<20 \%)$. UGA, however, improved the accuracy of forest, but a large part of grass was misidentified as forest (Figure 5(d)). In contrast, similar but better results were achieved by UPSO and ULPSO, with overall accuracies larger than 70\%. The Z-test results showed that ULPSO performed significantly better than $k$-means and UGA. The difference between UPSO and ULPSO, however, was not significant, despite the slight outperformance of ULPSO over UPSO (Table 4).

\subsection{Robustness and searching capacity of methods}

To evaluate the robustness of the proposed method, ULPSO as well as $k$-means, UGA, and UPSO were implemented 30 times with randomly generated initial population for each experiment. The average overall accuracy and Kappa coefficient were summarized in Table 5. It is observed from the table that the highest accuracy was obtained by ULPSO over all of the three experiments, followed by UPSO and UGA, while $k$-means demonstrated the worst. Besides, ULPSO worked much more stable (i.e. robust) than the other methods in view of its lower variance of accuracy (Table 5). To further compare the means of the classification results produced by methods, the student' $t$-test was performed on Kappa coefficient (Table 5). As shown in the table, the presented ULPSO beat the other methods in a statistically significant manner in the first two experiments. There was no significant difference among the three intelligent methods in the QuickBird experiment, despite ULPSO yielded the highest average accuracy (Table 5).

Table 5 is here

The average clustering metric values (number of repetition: 30 times) running versus numbers of iterations were calculated to investigate the searching capacity of methods. Figure 6 illustrated the corresponding results over the three experiments. It is shown that each method appeared similar patterns over the three experiments. In specific, UGA had the slowest rate of convergence, thus incapable of acquiring a lower cluster metric value, even if reaching the maximum number of iteration. In comparison, the fastest convergence rate was achieved by $k$-means, which becomes stable in the early stages (about 60 iterations) of the optimization; a similar convergence pattern was revealed by UPSO, maturing at about 100 iterations (Figure 6). However, both $k$-means and UPSO converged to high-level values of clustering metric, indicating they are subject to being fallen into local optimum prematurely. As for ULPSO, it not only maintains the same advantage of rapid convergence at the early optimization stage as UPSO, but also continuously and strongly optimizes (decreases) the 
value of objective function, which stabilizes at about 800 iterations (Figure 6). As a result, obviously lower clustering metric value was achieved by ULPSO, where Levy flight plays a crucial role in helping PSO jump out of local optima and eventually approach (or reach) the optimal solution.

Figure 6 is here

\subsection{Computational complexity analysis}

In this paper, the computational complexity was fairly compared since the same number of outer iterations (Miter) was utilized in each method. The complexity of the methods thus differs mainly from their numbers of inner loops. Consider $n, K$ are the number of population and the number of clusters, respectively. Among the four methods, $k$-means, with $K$ inner loops and Miter outer iterations, has the least complexity: $O(M i t e r K)$; for UGA, it can be expressed as $O\left(\operatorname{Miter}\left(n K+n p_{S}+1 / 2 n p_{c}+n p_{m}\right)\right)$, where $O(n K)$ is the complexity of objective function, while $O\left(n p_{s}\right), O\left(1 / 2 n p_{c}\right)$, and $O\left(n p_{m}\right)$ denote that of the three operators respectively: selection, cross, and mutation; UPSO and ULPSO have a relatively high complexity: $O(\operatorname{Miter}(n K+2 n))$ and $O(\operatorname{Miter}(n K+2 n+K))$, respectively. In comparison with UPSO, a little bit more complexity hold by ULPSO was noticed.

The practical computing time of classifications in the three experiments is listed in Table 6 . Note that all the three methods were implemented in a MATLAB environment, and run on a personal computer with 3.20-GHz CPU and 8.0-GB memory. As expected, the intelligent methods (UGA, UPSO, and ULPSO) required much more computation time than the simple $k$-means. This is because $k$-means uses only one candidate solution during each iteration of clustering, while 40 candidate solutions are simultaneously evolved for the three intelligent methods. The computational time is almost the same for UGA and UPSO, while a little bit more time is needed by ULPSO because of the computation of step length of Levy flight. But it is acceptable in view of the significant better results.

Table 6 is here

\section{Discussion}

In this paper, the problem of image clustering is transformed into an optimization problem. The number of variables contained in each candidate solution is fixed, i.e. the product $(D \times K)$ of dimension of attributes $(D)$ and number of clusters $(K)$. The aim of clustering is to find out proper variables in the solution space to form an optimal solution, so that the clustering metric (objective function) can be minimized. However, it is not a trivial task since image clustering usually has a huge solution space. This paper is to establish an effective and reliable searching mechanism in the space.

The proposed ULPSO proves superior to traditional $k$-means, UGA, and UPSO over all of the three experiments. In UGA, candidate solutions were randomly crossed and mutated (Yen et al. 1998), without further seeking candidates around the current optimum. Achieving the 
slowest convergence rate (Figure 6), UGA is therefore in short of the necessary exploitation capacity for handling complex remote sensing image. As for UPSO, the evolution is carried out by considering the global (i.e. the whole swarm) optimal solution and personal (i.e. an individual particle) optima (Equation (6)). UPSO hence owns a high exploitation capacity (Kusetogullari et al. 2015), which well explains the reason of its rapid convergence (Figure 6). Yet, such an evolving strategy would make all individual particles resemble each other (Arani et al. 2013), thus having difficulty maintaining the diversity of population. As a consequence, the swarm tends to be premature (i.e. trapping on local optima) in the early evolution stage (Figure 6) due to the lack of exploration capacity (Kaveh and Zolghadr 2014).

Satisfactorily, by adopting Levy flight, the proposed ULPSO method possesses not only the inherent exploitation capacity (like UPSO) but also a powerful exploration capacity, as illustrated by Figure 6. The searching step produced by Levy flight can differ in length, thus enabling to offer long steps with a certain probability (Yang 2010). The worst particle (with the lowest fitness), whose new position was assigned by Levy flight in each iteration, can then be served as a scout particle to pioneer new spaces. Such a searching strategy guarantees the local exploitation capability of the population; at the same time, the particles are able to travel throughout the whole solution space, thus providing a global searching ability. The integration of Levy flight and the PSO would provide a complementary behavior (exploration and exploitation). Thanks to this novel searching strategy (the major contribution of this research), significant better results were achieved by ULPSO in comparison with UPSO.

Some previous researches enhanced the PSO-based image clustering by integrating other algorithms (e.g. decision-making; Naeini et al. 2014) or classifiers (e.g. SVM; Venkatalakshmi and Shalinie 2005). There are also some studies employing PSO to optimize other clustering algorithms (e.g. fuzzy c-means; Samadzadegan and Naeini 2011; Niazmardi et al. 2012). The basic searching strategy of PSO, however, has received little attention in the literature. Toward this end, Levy flight was introduced to enhance the exploration capacity of PSO, i.e. to fundamentally improve the algorithm from the underlying searching mechanism. That's why different types of remote sensing images adopted in this study (including TM, FLC and QuickBird) were effectively handled by ULPSO. In fact, the proposed method is universally applicable, not only to different kinds of remotely sensed images but also to a variety of application programs of geosciences and remote sensing, such as image band selection (Su et al. 2014), land use allocation (Liu et al. 2013), and urban growth modeling (Feng et al. 2011).

We note that a few early efforts had been made to improve PSO by Levy flight for function optimization, and presented the corresponding methods (LFPSO, Hakli and Uguz 2014; PSOLF, Jensi and Jiji 2016). However, the searching strategies employed in these methods were in short of balanced exploitation and exploration capabilities, thus having difficulty addressing the complex problem of image clustering. For LFPSO, a particle's position is updated by Levy flight, only if it cannot be improved after reaching a predefined searching time limitation. Such a searching mechanism may reduce the exploration capacity of LFPSO, since those continuously evolved particles with low fitness could still survive after a large number of iteration. This leads to a waste of valuable searching sources. Besides, it is hard to determine a proper value of searching time limitation. As for PSOLF, positions of as many as one-half particles are regenerated by Levy flight at each iteration, leading to an 
intense fluctuation of the population. This may substantially weaken the exploitation capacity of the method. The searching capacity of the four PSO-based methods (i.e. UPSO, LFPSO, PSOLF, and ULPSO) was tested in the TM experiment, with each method performed 10 times. As illustrated by Figure 7 in which variations of the average clustering metric value for each method were compared, PSOLF performed the worst, followed by UPSO and PSOLF; the proposed ULPSO achieved the best result thanks to its balanced and powerful exploitation and exploration capabilities as discussed previously. Figure 8 further demonstrates that the proposed method achieved better and more stable classification results than the other methods.

Figure 7 is here

\section{Conclusions}

Unsupervised remote sensing image classification, that typically has very large solution spaces, belongs to the family of NP-hard problems. Traditional $k$-means and common intelligent methods (such as genetic algorithm) tend to become trapped on local optima due to their limited exploitation or exploration capacities. In this paper, we develop a novel ULPSO methodology in which a new searching strategy with Levy flight is adopted for unsupervised image classification. Due to its powerful exploitation ability, ULPSO converged very rapidly at the early optimization stage like UPSO. More importantly, it could jump out of local optima and continuously approach (or reach) the optimal solution, thanks to the relatively powerful exploration capacity benefiting from Levy flight. ULPSO has been shown to be superior to the other methods over all of the experiments. The proposed method is, therefore, suggested to be an alternative for unsupervised remote sensing image classification.

Although ULPSO achieved encouraging results, we notice that it is still difficult to yield satisfactory image classification result relying on spectral information purely (the 'salt-and-pepper' phenomenon), especially for the very high resolution (VHR) image (Figure 5). Incorporating spatial information of image into ULPSO deserves much more investigation. In addition, we would like to explore the possibility of developing an automatic clustering method based on the presented method. In that case, intra criterion (clustering metric in our experiments) as well as inter criterion should be simultaneously considered in the process of optimization.

\section{Acknowledgements}

This research was supported by the National Natural Science Foundation of China (grant number: 41301465) and the Scientific and Technological Development Program of Jilin Province (grant number: 20170520087JH).

\section{References}

Arani, B. O., P. Mirzabeygi and M. S. Panahi. 2013. "An improved PSO algorithm with a territorial diversity-preserving scheme and enhanced exploration-exploitation balance." Swarm and Evolutionary Computation 11: 1-15. doi:10.1016/j.swevo.2012.12.004. 
Bandyopadhyay, S. and U. Maulik. 2002. "Genetic clustering for automatic evolution of clusters and application to image classification." Pattern Recognition 35: 1197-1208. doi:Pii S0031-3203(01)00108-X.

Bonan, G. B. 1997. "Effects of land use on the climate of the United States." Climatic Change 37: 449-486. doi: 10.1023/A:1005305708775.

Bounoua, L., R. Defries, G. J. Collatz, P. Sellers and H. Khan. 2002. "Effects of land cover conversion on surface climate." Climatic Change 52: 29-64.doi: 10.1023/A:1013051420309.

Camps-Valls, G., T. V. Bandos and D. Y. Zhou. 2007. "Semi-supervised graph-based hyperspectral image classification." Ieee Transactions on Geoscience and Remote Sensing 45: 3044-3054. doi:10.1109/Tgrs.2007.895416.

Chang, Y. L., J. P. Fang, L. N. Chang, J. A. Benediktsson, H. A. Ren and K. S. Chen. 2009. "Band Selection for Hyperspectral Images Based on Parallel Particle Swarm Optimization Schemes." 2009 Ieee International Geoscience and Remote Sensing Symposium, Vols 1-5: 3509-3512.

Das, P. K., H. S. Behera and B. K. Panigrahi. 2016. "A hybridization of an improved particle swarm optimization and gravitational search algorithm for multi-robot path planning." Swarm and Evolutionary Computation 28: 14-28. doi:10.1016/j.swevo.2015.10.011.

De Colstoun, E. C. B. and C. L. Walthall. 2006. "Improving global scale land cover classifications with multi-directional POLDER data and a decision tree classifier." Remote Sensing of Environment 100: 474-485. doi:10.1016/j.rse.2005.11.003.

Deng, X. Z., Q. L. Shi, Q. Zhang, C. C. Shi and F. Yin. 2015. "Impacts of land use and land cover changes on surface energy and water balance in the Heihe River Basin of China, 2000-2010." Physics and Chemistry of the Earth 79-82: 2-10. doi:10.1016/j.pce.2015.01.002.

Duda, T. and M. Canty. 2002. "Unsupervised classification of satellite imagery: choosing a good algorithm." International Journal of Remote Sensing 23: 2193-2212. doi: 10.1080/01430060110078467.

Edwards, A. M., R. A. Phillips, N. W. Watkins, M.P. Freeman, E.J. Murphy, V. Afanasyev, et al. 2007. "Revisiting Levy flight search patterns of wandering albatrosses, bumblebees and deer." Nature 449: 1044-1045. doi:10.1038/nature06199.

Feng, Y. J., Y. Liu, X. H. Tong, M. L. Liu and S. S. Deng. 2011. "Modeling dynamic urban growth using cellular automata and particle swarm optimization rules." Landscape and Urban Planning 102: 188-196. doi:10.1016/j.landurbplan.2011.04.004.

Foody, G. M. 2002. "Status of land cover classification accuracy assessment." Remote Sensing of Environment 80: 185-201. doi:Pii S0034-4257(01)00295-4.

Gong, P., J. Wang, L. Yu, Y.C. Zhao, Y.Y. Zhao, L. Liang, Z.G. Niu, X. Huang, et al. 2013. "Finer resolution observation and monitoring of global land cover: first mapping results with Landsat TM and ETM+ data." International Journal of Remote Sensing 34: 2607-2654. doi:10.1080/ 01431161.2012 .748992 .

Hakli, H. and H. Uguz. 2014. "A novel particle swarm optimization algorithm with Levy flight." Applied Soft Computing 23: 333-345. doi:10.1016/j.asoc.2014.06.034.

Huang, X. and L. P. Zhang. 2010. "Comparison of Vector Stacking, Multi-SVMs Fuzzy Output, and Multi-SVMs Voting Methods for Multiscale VHR Urban Mapping." Ieee Geoscience and Remote Sensing Letters 7: 261-265. doi:10.1109/Lgrs.2009.2032563.

Jensi, R. and G. W. Jiji. 2016. "An enhanced particle swarm optimization with levy flight for global optimization." Applied Soft Computing 43: 248-261.doi:10.1016/j.asoc.2016.02.018. 
Jung, M., K. Henkel, M. Herold and G. Churkina. 2006. "Exploiting synergies of global land cover products for carbon cycle modeling." Remote Sensing of Environment 101: 534-553. doi:10.1016/j.rse.2006.01.020.

Karaboga, D. and B. Akay. 2009. "A comparative study of Artificial Bee Colony algorithm." Applied Mathematics And Computation 214: 108-132.doi:10.1016/j.amc.2009.03.090.

Kaveh, A. and A. Zolghadr. 2014. "Democratic PSO for truss layout and size optimization with frequency constraints." Computers \& Structures 130: 10-21.doi:10.1016/j.compstruc.2013.09.002.

Kennedy, J. and R. Eberhart. 1995. "Particle swarm optimization." 1995 Ieee International Conference on Neural Networks Proceedings, Vols 1-6: 1942-1948. doi: 10.1109/Icnn.1995.488968.

Kusetogullari, H., A. Yavariabdi and T. Celik. 2015. "Unsupervised Change Detection in Multitemporal Multispectral Satellite Images Using Parallel Particle Swarm Optimization." Ieee Journal of Selected Topics in Applied Earth Observations and Remote Sensing 8: 2151-2164. doi:10.1109/Jstars.2015.2427274.

Li, H. P., S. Q. Zhang, X. H. Ding, C. Zhang and P. Dale. 2016a. "Performance Evaluation of Cluster Validity Indices (CVIs) on Multi/Hyperspectral Remote Sensing Datasets." Remote Sensing 8: 295. doi:10.3390/rs8040295.

Li, H. P., S. Q. Zhang, X. H. Ding, C. Zhang and R. Cropp. 2016b. "A novel unsupervised bee colony optimization (UBCO) method for remote-sensing image classification: a case study in a heterogeneous marsh area." International Journal of Remote Sensing 37: 5726-5748. doi:10.1080/01431161.2016.1246771.

Li, H. P., S. Q. Zhang, Y. Sun and J. Gao. 2011. "Land cover classification with multi-source data using evidential reasoning approach." Chinese Geographical Science 21: 312-321. doi: 10.1007/s11769-011-0465-1.

Liu, X. P., J. P. Ou, X. Li and B. Ai. 2013. "Combining system dynamics and hybrid particle swarm optimization for land use allocation." Ecological Modelling 257: 11-24. doi:10.1016/j.ecolmodel.2013.02.027.

Liu, X. P., X. Li, X. J. Peng, H. B. Li and J. Q. He. 2008. "Swarm intelligence for classification of remote sensing data." Science in China Series D-Earth Sciences 51: 79-87. doi:10.1007/s11430-007-0133-6.

Loveland, T. R., B. C. Reed, J. F. Brown, D. O. Ohlen, Z. Zhu, L. Yang and J. W. Merchant. 2000. "Development of a global land cover characteristics database and IGBP DISCover from $1 \mathrm{~km}$ AVHRR data." International Journal of Remote Sensing 21: 1303-1330. doi: 10.1080/014311600210191.

Mantegna, R. N. 1994. "Fast, Accurate Algorithm for Numerical-Simulation of Levy Stable Stochastic-Processes." Physical Review E 49: 4677-4683. doi: 10.1103/PhysRevE.49.4677.

Masoomi, Z., M. S. Mesgari and M. Hamrah. 2013. "Allocation of urban land uses by Multi-Objective Particle Swarm Optimization algorithm." International Journal of Geographical Information Science 27: 542-566. doi:10.1080/13658816.2012.698016.

Maulik, U. and S. Bandyopadhyay. 2000. "Genetic algorithm-based clustering technique." Pattern Recognition 33: 1455-1465. doi: 10.1016/S0031-3203(99)00137-5.

Mukhopadhyay, S., P. Mandal, T. Pal and J. K. Mandal. 2015. "Image Clustering Based on Different Length Particle Swarm Optimization (DPSO)." Proceedings of the 3rd International Conference on Frontiers of Intelligent Computing: Theory and Applications (Ficta) 2014, Vol 1 327: 711-718. doi:10.1007/978-3-319-11933-5_80. 
Naeini, A. A., S. Homayouni and M. Saadatseresht. 2014. "Improving the Dynamic Clustering of Hyperspectral Data Based on the Integration of Swarm Optimization and Decision Analysis." Ieee Journal of Selected Topics in Applied Earth Observations and Remote Sensing 7: 2161-2173. doi:10.1109/Jstars.2014.2307579.

Nayak, S. K., K. R. Krishnanand, B. K. Panigrahi and P. K. Rout. 2009. "Application of Artificial Bee Colony to Economic Load Dispatch Problem with Ramp Rate Limits and Prohibited Operating Zones." 2009 World Congress on Nature \& Biologically Inspired Computing (Nabic 2009): 1236-1241.

Niazmardi, S., A. A. Naeini, S. Homayouni, A. Safari and F. Samadzadegan. 2012. "Particle swarm optimization of kernel-based fuzzy c-means for hyperspectral data clustering." Journal of Applied Remote Sensing 6. doi:Artn06360110.1117/1.Jrs.6.063601.

Omran, M., A. P. Engelbrecht and A. Salman. 2005. "Particle swarm optimization method for image clustering." International Journal of Pattern Recognition and Artificial Intelligence 19: 297-321. doi: 10.1142/S0218001405004083.

Paoli, A., F. Melgani and E. Pasolli. 2009. "Clustering of Hyperspectral Images Based on Multiobjective Particle Swarm Optimization." Ieee Transactions on Geoscience and Remote Sensing 47: 4175-4188. doi:10.1109/TGRS.2009.2023666.

Samadzadegan, F. and A. A. Naeini. 2011. "Fuzzy clustering of hyperspectral data based on particle swarm optimization." 2011 3rd Workshop on Hyperspectral Image and Signal Processing: Evolution in Remote Sensing (WHISPERS), 1-4.

Senthilnath J., S. Kulkarni, D.R. Raghuram, M. Sudhindra, S.N. Omkar, V. Das and V. Mani. 2016, “A Novel Harmony Search Based Approach for Clustering Problems”. International Journal of Swarm Intelligence, 2: 66 - 86. doi: 10.1504/IJSI.2016.077434.

Senthilnath, J., V. Das, S. N. Omkar and V. Mani. 2013. "Clustering using Levy Flight Cuckoo Search." Proceedings of Seventh International Conference on Bio-Inspired Computing: Theories and Applications (Bic-Ta 2012), 202: 65-75. doi:10.1007/978-81-322-1041-2_6.

Seo, J. H., C. H. Im, C. G. Heo, J. K. Kim, H. K. Jung and C. G. Lee. 2006. "Multimodal function optimization based on particle swarm optimization." Ieee Transactions on Magnetics 42: 1095-1098. doi:10.1109/TMAG.2006.871568.

Srinivasan, D. and T.H. Seow. 2003. "Particle Swarm Inspired Evolutionary Algorithm (PS-EA) for Multiobjective Optimization Problem." Proceedings of the 2003 Congress on Evolutionary Computation, Canberra, Australia, 2292-2297.

Su, H. J., Q. Du, G. S. Chen and P. J. Du. 2014. "Optimized Hyperspectral Band Selection Using Particle Swarm Optimization." Ieee Journal of Selected Topics in Applied Earth Observations and Remote Sensing 7: 2659-2670. doi:10.1109/JSTARS.2014.2312539.

Tadjudin, S. and D. A. Landgrebe. 2000. "Robust parameter estimation for mixture model." Ieee Transactions on Geoscience and Remote Sensing 38: 439-445. doi:10.1109/36.823939.

Tseng, C. T. and C. J. Liao. 2008. "A discrete particle swarm optimization for lot-streaming flowshop scheduling problem." European Journal Of Operational Research 191: 360-373. doi:10.1016/j.ejor.2007.08.030.

Verburg, P. H., K. Neumann and L. Nol. 2011. "Challenges in using land use and land cover data for global change studies." Global Change Biology 17: 974-989. doi:10.1111/j.1365-2486.2010.02307.x.

Wang, L., W. P. Sousa, P. Gong and G. S. Biging. 2004. "Comparison of IKONOS and QuickBird 
images for mapping mangrove species on the Caribbean coast of Panama." Remote Sensing of Environment 91: 432-440. doi:10.1016/j.rse.2004.04.005.

Wang, Q. M., L. G. Wang and D. F. Liu. 2012. "Particle swarm optimization-based sub-pixel mapping for remote-sensing imagery." International Journal of Remote Sensing 33: 6480-6496. doi:10.1080/01431161.2012.690541.

Wilkinson, G. G. 2005. "Results and implications of a study of fifteen years of satellite image classification experiments." Ieee Transactions on Geoscience and Remote Sensing 43: 433-440. doi:10.1109/TGRS.2004.837325.

Wong, M. T., X. J. He and W. C. Yeh. 2011. "Image Clustering Using Particle Swarm Optimization." 2011 Ieee Congress on Evolutionary Computation (Cec): 262-268.

Xu, K., W. Yang, G. Liu and H. Sun. 2013. "Unsupervised Satellite Image Classification Using Markov Field Topic Model." Ieee Geoscience and Remote Sensing Letters 10: 130-134. doi:10.1109/LGRS.2012.2194770.

Xu, Y. F. and S. L. Zhang. 2009. "Fuzzy Particle Swarm Clustering of Infrared Images." Icic 2009: Second International Conference on Information and Computing Science, Vol 2, Proceedings: 122-124.doi:10.1109/Icic.2009.139.

Yang, F. Q., T. E. L. Sun and C. H. Zhang. 2009. "An efficient hybrid data clustering method based on K-harmonic means and Particle Swarm Optimization." Expert Systems with Applications 36: 9847-9852. doi:10.1016/j.eswa.2009.02.003.

Yang, X. S. 2010. Nature-Inspired Metaheuristic Algorithms. Luniver Press, $2^{\text {nd }}$ edition.

Yang, X. S. and S. Deb. 2013. "Multiobjective cuckoo search for design optimization." Computers \& Operations Research 40: 1616-1624. doi:10.1016/j.cor.2011.09.026.

Yen, J., J. C. Liao, B. J. Lee and D. Randolph. 1998. "A hybrid approach to modeling metabolic systems using a genetic algorithm and simplex method." Ieee Transactions on Systems Man and Cybernetics Part B-Cybernetics 28: 173-191. doi:10.1109/3477.662758.

Yildirim, A. 2014. "Unsupervised classification of multispectral Landsat images with multidimensional particle swarm optimization." International Journal of Remote Sensing 35: 1217-1243. doi:10.1080/01431161.2013.877617.

Yu, P., A. K. Qin and D. A. Clausi. 2012. "Unsupervised Polarimetric SAR Image Segmentation and Classification Using Region Growing With Edge Penalty." Ieee Transactions on Geoscience and Remote Sensing 50: 1302-1317. doi:10.1109/TGRS.2011.2164085.

Yu, Y. F., G. Li and C. Xu. 2013. "An Improved Particle Swarm Optimization Algorithm." Frontiers of Manufacturing Science and Measuring Technology Iii, Pts 1-3 401: 1328-1335. doi:10.4028/www.scientific.net/AMM.401-403.1328.

Zhong, Y. F., L. P. Zhang, B. Huang and P. X. Li. 2006. "An unsupervised artificial immune classifier for multi/hyperspectral remote sensing imagery." Ieee Transactions on Geoscience and Remote Sensing 44: 420-431. doi:10.1109/TGRS.2005.861548. 